\title{
Introduction to the Ecology of Literacy Diversity in the New Regimes of Information
}

\begin{abstract}
Promoting discourses to explore the variety of literacies, opens platforms to engage with the meaning of 'literacy'. No longer is it delimited to basic information literacy, but it has evolved to include media and information literacy (MIL), digital, gaming, and Artificial Intelligence (AI) literacies amongst others. Hence the very suitable umbrella term of an ecology of literacies for this volume.
\end{abstract}

In general, being literate enables one to communicate, create, and collaborate. These skills allow one to integrate basic to complex literacies to suit the requirements within education, employment, citizen participation and social interaction ${ }^{1}$. In terms of future considerations, NetLiteracy argues that AI Literacy will improve critical thinking skills and empower people to make use of AI to make informed decisions about education, professional and personal development ${ }^{2}$. However, being literate and engaging with Information and Communication Technologies (ICTs) brings to the fore information ethical considerations of emerging technologies.

In 2020 UNESCO appointed an international expert group to draft global recommendation on the ethics of $\mathrm{AI}^{3}$. The Director-General of UNESCO declared that "It is our responsibility to lead a universal enlightened debate in order to enter this new era with our eyes wide open, without sacrificing our values, and establish a common global foundation of ethical principles for artificial intelligence". In the draft recommendation of the Ethics of AI, there is regular mention of literacy and stakeholder collaboration needed to ensure these requisite competencies. In the section on Principles, literacy is coupled with awareness (paragraph 32): Public awareness and understanding of AI technologies and the value of data should be promoted through education, public campaigns and training to ensure effective public participation so that citizens can take informed decisions about their use of AI systems. Children should be protected from reasonably foreseeable harms arising from AI systems, should have access to such systems through education and training, and children should not be disempowered by their interaction with AI systems. This is supported by Policy Action 3 - addressing the social and economic impact of AI. Paragraph 53 indicates that Member States should work with international organizations, private and nongovernmental entities to provide adequate AI literacy education to the public especially in LMICS (low-to-middle-income-countries) in order to reduce the digital divide and digital access inequalities resulting from the wide adoption of AI systems. And finally, the recommendations state in Policy Actions 5 - promoting AI ethics education and awareness (paragraph 68), that Member States should promote the acquisition of 'prerequisite skills' for AI education, such as basic literacy, numeracy, and coding skills, especially in countries where there are notable gaps in the education of these skills.

Being illiterate precludes one to partake in the job market, hence the necessity of literacy integration in all levels of education to promote life-long learning ${ }^{4}$. An ecology of literacy - coupled with information ethics challenges society to prioritise its integration in discourses. Consequently, this volume celebrates the diversity of literacy not only in content, but also in form. Composed in English, Portuguese and Spanish, the volume includes articles, interviews, and a book review. We invite you to explore this space with us and the authors.

\section{Sincerely Yours}

Rachel Fischer

\footnotetext{
${ }^{1}$ Media and Information Literacy Curriculum for Teachers | United Nations Educational, Scientific and Cultural Organization, 2011. Available: http://www.unesco.org/new/en/communication-and-information/resources/publications-and-communicationmaterials/publications/full-list/media-and-information-literacy-curriculum-for-teachers/

${ }^{2}$ NetLiteracy. AI literacy. n.d. Available: https://www.netliteracy.org/ai-literacy/

${ }^{3}$ UNESCO. UNESCO appoints international expert group to draft global recommendation on the ethics of AI. 2020. Available: https://en.unesco.org/news/unesco-appoints-international-expert-group-draft-global-recommendation-ethics-ai

${ }^{4}$ Yeo, C. Why we need AI literacy. 2020. Available: https://medium.com/fair-bytes/why-we-need-ai-literacy-716d5a63ecc3
} 